\title{
Diophantine Equations and the Freeness of Möbius Groups
}

\author{
Marin Gutan \\ Laboratoire de Mathématiques, Université Blaise-Pascal, Clermont-Ferrand, France \\ Email: marin.gutan@math.univ-bpclermont.fr
}

Received 16 March 2014; revised 16 April 2014; accepted 23 April 2014

Copyright (C 2014 by author and Scientific Research Publishing Inc.

This work is licensed under the Creative Commons Attribution International License (CC BY).

http://creativecommons.org/licenses/by/4.0/

(c) (i) Open Access

\section{Abstract}

Let $p$ and $q$ be two fixed non zero integers verifying the condition $\operatorname{gcd}(p, q)=1$. We check solutions in non zero integers $a_{1}, b_{1}, a_{2}, b_{2}$ and $a_{3}$ for the following Diophantine equations: (B1) $\left(a_{1}+a_{2}\right) q^{2}+a_{1} b_{1} a_{2} p^{2}=0$ $\left(a_{1}+a_{2}+a_{3}\right) q^{4}+\left(a_{1} b_{1} a_{2}+a_{1} b_{1} a_{3}+a_{1} b_{2} a_{3}+a_{2} b_{2} a_{3}\right) q^{2} p^{2}+a_{1} b_{1} a_{2} b_{2} a_{3} p^{4}=0$. The equations (B1) and (B2) were considered by R.C. Lyndon and J.L. Ullman in [1] and A.F. Beardon in [2] in connection with the freeness of the Möbius group $G_{\lambda}$ generated by two matrices of $\mathcal{S} \mathcal{L}_{2}(\mathbb{C})$, namely $A=\left(\begin{array}{ll}1 & \lambda \\ 0 & 1\end{array}\right)$ and $B=\left(\begin{array}{ll}1 & 0 \\ \lambda & 1\end{array}\right)$ where $\lambda=\frac{p}{q}$. They proved that if one of the equations (B1) or (B2) has solutions in non zero integers then the group $G_{\lambda}$ is not free. We give algorithms to decide if these equations admit solutions. We obtain an arithmetical criteria on $p$ and $q$ for which (B1) admits solutions. We show that for all $p$ and $q$ the equations $(B 1)$ and $(B 2)$ have only a finite number of solutions.

\section{Keywords}

Diophantine Equation, Möbius Groups, Free Group

\section{Introduction}

Let $k$ and $d$ be two positive integers with $d \geq 2$ and $A_{1}, \cdots, A_{k}$ be matrices of the group $\mathcal{G} \mathcal{L}_{d}(\mathbb{C})$. 
Denote $\mathcal{G} p\left(A_{1}, \cdots, A_{k}\right)$ the group, respectively $\operatorname{Sgp}\left(A_{1}, \cdots, A_{k}\right)$ the semigroup, generated by the matrices $A_{1}, \cdots, A_{k}$.

The following problem $\mathfrak{P}$ has been studied in several papers:

- Instance: $A_{1}, \cdots, A_{k} \in \mathcal{G} \mathcal{L}_{d}(\mathbb{C})$

- Question: $\mathcal{G} p\left(A_{1}, \cdots, A_{k}\right)$ or $\mathcal{S} g p\left(A_{1}, \cdots, A_{k}\right)$ are they free with $A_{1}, \cdots, A_{k}$ as generators?

Recall that in 1991 D. Klarner, J.-C. Birget and W. Satterfield in [3] proved that if $d \geq 3$ then the problem $\mathfrak{P}$ is not decidable. Moreover in $1999 \mathrm{~J}$. Cassaigne, T. Harju and J. Karhümaki in [4] proved that the same result is true if we suppose that all the matrices $A_{1}, \cdots, A_{k}$ are lower triangular.

The case $d=2$ is open and seems difficult. In [5] and [6] results concerning the freeness of the semigroups and groups generated by two matrices are established. In this paper we are studying this problem restricted to the case of Möbius groups.

Let $\lambda \in \mathbb{C}^{*}$ and $\tau=\lambda^{2}$. The Möbius group $G_{\lambda}$ is the subgroup of $\mathcal{S L}_{2}(\mathbb{C})$ generated by $A=\left(\begin{array}{ll}1 & \lambda \\ 0 & 1\end{array}\right)$ and $B=\left(\begin{array}{ll}1 & 0 \\ \lambda & 1\end{array}\right)$.

The problem of characterization of the set of complex values of $\lambda$ or $\tau$ for which the group $G_{\lambda}$ is free, was studied in several papers. Thus in [1] it is proved that if $\lambda$ is transcendental or $|\lambda| \geq 2$ then $G_{\lambda}$ is free.

R.C. Lyndon and J.L. Ullman in [1] remarked that $G_{\lambda}$ is not free if and only if there exists a word $w=a_{1} b_{1} a_{2} b_{2} \cdots a_{n-1} b_{n-1} a_{n}$ whose $2 n-1$ letters are non zero integers so that the product of the powers of matrices $M_{\lambda}(w)=A^{a_{1}} B^{b_{1}} A^{a_{2}} B^{b_{2}} \cdots A^{a_{n-1}} B^{b_{n-1}} A^{a_{n}}$ is a lower triangular matrix. The element in the right upper corner of the matrix $M_{\lambda}(w)$ is of the form $\lambda \mathcal{Q}_{\tau}(w)$ where $\mathcal{Q}_{\tau}(w)=\sum_{j=0}^{n-1} c_{j} \tau^{j}$ is a polynomial in $\tau$ of degree $n-1$ with coefficients $c_{j}$. Moreover $c_{j}$ are polynomials with integers coefficients in the variables $a_{1}, b_{1}, a_{2}, b_{2}, \cdots, a_{n}$.

Results concerning the set of algebraic values of $\lambda$ or $\tau$ for which the group $G_{\lambda}$ is not free were obtained in [1] [2] [7]-[11].

Deciding if for $\tau \in \mathbb{Q} \cap] 0,4\left[\right.$ the group $G_{\lambda}$ is not free seems very difficult. Let us recall some important results in this direction.

The group $G_{\lambda}$ is not free if $\lambda$ belongs to one of the following sets: $A=\left\{\frac{1}{n} \mid n \in \mathbb{Z}^{*}\right\}$,

$B=\left\{\frac{p}{k p+1} \mid p, k \in \mathbb{N}^{*}\right\}, \quad C=\left\{\frac{m+n}{m n} \mid m, n \in \mathbb{N}^{*}, m+n>1\right\} \quad$ (see [1] [2] [7] [8] [10] [11]).

In this paper we check if for a given $\tau \in \mathbb{Q} \cap] 0,4[$ there exists a non trivial word of non zero integers $w=a_{1} b_{1} a_{2} b_{2} \cdots a_{n-1} b_{n-1} a_{n}$ such that $Q_{\tau}(w)=0$.

The main results of our paper concern the freeness of Möbius groups:

- We prove that if the length of $w$ is small then the problem is decidable (cases $n=2$ and $n=3$ ) (see Theorems 1, 2 and 3).

- We give algorithms which solve the problem for $n \in\{2,3\} \quad$ (see Corollary 1 and the proof of Theorem 3). Moreover, we give an arithmetical criteria for this problem when $n=2$ (see 2 of Theorem 1).

- We give a lower bound numerical function $l$ defined from $\mathbb{Q} \cap] 2,4\left[\right.$ to $\mathbb{N}^{*}$, increasing and unbounded, such that for each $\tau \in \mathbb{Q} \cap] 2,4\left[\right.$, if $M_{\tau}(w)$ is a lower triangular matrix then the length of $w$ is bigger than $I(\tau)$ (see Theorem 4 and Corollary 3).

As proved by A.F. Beardon ([2]) in these two cases $n \in\{2,3\}$ we have to find solutions for the equations $(B 1)$ and (B2). In fact in our paper we consider and study two more general equations:

(B'1) $\left(a_{1}+a_{2}\right) q+a_{1} b_{1} a_{2} p=0$

(B'2) $\left(a_{1}+a_{2}+a_{3}\right) q^{2}+\left(a_{1} b_{1} a_{2}+a_{1} b_{1} a_{3}+a_{1} b_{2} a_{3}+a_{2} b_{2} a_{3}\right) q p+a_{1} b_{1} a_{2} b_{2} a_{3} p^{2}=0$.

\section{Sequences of Polynomials Associated to Matrices}

In this section, we study the properties of some sequences of polynomials in a fixed $\tau=\lambda^{2}$ associated to matrices of the group $G_{\lambda}$. 
We consider $\left((\mathbb{Z} \backslash\{0\})^{*}, \cdot\right)$ the free monoid of words on non zero integers with the concatenation operation. We denote by $\epsilon$ the empty word of the free monoid $(\mathbb{Z} \backslash\{0\})^{*}$ and a non empty word $w \in(\mathbb{Z} \backslash\{0\})^{*}$ by $w=k_{1} k_{2} k_{3} \cdots k_{n}$, where $k_{1}, \cdots, k_{n}$ are non zero integers. Then $n$ is called the length of $w$ and is denoted by $|w|$. The reversal of a word $w=k_{1} k_{2} k_{3} \cdots k_{n}$ is $w^{\sim}=k_{n} \cdots k_{3} k_{2} k_{1}$ and the opposite of $w$ is $-w=\left(-k_{1}\right)\left(-k_{2}\right)\left(-k_{3}\right) \cdots\left(-k_{n}\right)$.

For every word $w=a_{1} b_{1} a_{2} b_{2} \cdots a_{n-1} b_{n-1} a_{n}$ of $(\mathbb{Z} \backslash\{0\})^{*}$ of length $|w|=2 n-1$ we consider the matrix product

$$
M_{\lambda}(w)=A^{a_{1}} B^{b_{1}} A^{a_{2}} B^{b_{2}} \cdots A^{a_{n-1}} B^{b_{n-1}} A^{a_{n}} .
$$

For instance, for $a_{1}, b_{1}, a_{2}$ non zero integers we have:

$$
M_{\lambda}\left(a_{1}\right)=\left(\begin{array}{cc}
1 & a_{1} \lambda \\
0 & 1
\end{array}\right) \text { and } M_{\lambda}\left(a_{1} b_{1} a_{2}\right)=\left(\begin{array}{cc}
1+a_{1} b_{1} \tau & \lambda\left(a_{1}+a_{2}+a_{1} b_{1} a_{2} \tau\right) \\
b_{1} \lambda & 1+a_{2} b_{1} \tau
\end{array}\right) .
$$

We use the notation:

$$
M_{\lambda}(w)=\left(\begin{array}{cc}
\mathcal{P}_{\tau}(w) & \lambda \mathcal{Q}_{\tau}(w) \\
\lambda \mathcal{R}_{\tau}(w) & \mathcal{S}_{\tau}(w)
\end{array}\right)
$$

We remark that $\mathcal{P}_{\tau}(w), \mathcal{Q}_{\tau}(w), \mathcal{R}_{\tau}(w)$ and $\mathcal{S}_{\tau}(w)$ are polynomials in $\tau$ with coefficients in $\mathbb{Z}$. We also have $(-\omega)^{\sim}=-\left(\omega^{\sim}\right)$ and $\left[M_{\lambda}(\omega)\right]^{-1}=M_{\lambda}\left((-\omega)^{\sim}\right)$

If $\mathcal{T} \in\{\mathcal{P}, \mathcal{S}\} \quad$ then $\quad \mathcal{T}_{\tau}(-w)=\mathcal{T}_{\tau}(w) \quad$ and $\quad$ if $\quad \mathcal{T} \in\{\mathcal{Q}, \mathcal{R}\} \quad$ then $\quad \mathcal{T}_{\tau}(-w)=-\mathcal{T}_{\tau}(w)$. Also $\mathcal{P}(w)=\mathcal{S}\left(w^{\sim}\right)$ and if $\mathcal{T} \in\{\mathcal{Q}, \mathcal{R}\}$ then $\mathcal{T}_{\tau}(w)=\mathcal{T}_{\tau}\left(w^{\sim}\right)$.

We use the notation $M_{\lambda}(w)=\left(\begin{array}{ll}* & 0 \\ * & *\end{array}\right)$ to indicate that $M_{\tau}(w)$ is a lower triangular matrix or that $\mathcal{Q}_{\tau}(w)=0$.

From now on, in order to simplify the notation we write:

$$
\left(\begin{array}{cc}
P_{n} & \lambda Q_{n} \\
\lambda R_{n} & S_{n}
\end{array}\right)=\left(\begin{array}{cc}
\mathcal{P}_{\tau}(w) & \lambda \mathcal{Q}_{\tau}(w) \\
\lambda \mathcal{R}_{\tau}(w) & \mathcal{S}_{\tau}(w)
\end{array}\right)
$$

For instance, $P_{n}$ is an abbreviation for the polynomial in $\tau$ with parameters $a_{1}, b_{1}, a_{2}, b_{2}, \cdots, a_{n-1}, b_{n-1}, a_{n}$ defined by:

$$
P_{n}\left(a_{1}, b_{1}, a_{2}, b_{2}, \cdots, a_{n-1}, b_{n-1}, a_{n}\right)=\mathcal{P}_{\tau}\left(a_{1} b_{1} a_{2} b_{2} \cdots a_{n-1} b_{n-1} a_{n}\right) .
$$

Using the fact that $A, B \in \mathcal{S L}_{2}(\mathbb{C})$ we have:

$$
P_{n} S_{n}-\tau Q_{n} R_{n}=1
$$

The sequences of polynomials in $\tau,\left(P_{n}\right)_{n \geq 1},\left(Q_{n}\right)_{n \geq 1},\left(R_{n}\right)_{n \geq 1}$ and $\left(S_{n}\right)_{n \geq 1}$ verify the following relations:

$$
\begin{gathered}
\left(\begin{array}{ll}
P_{1} & Q_{1} \\
R_{1} & S_{1}
\end{array}\right)=\left(\begin{array}{cc}
1 & a_{1} \\
0 & 1
\end{array}\right) \\
\left(\begin{array}{cc}
P_{2} & Q_{2} \\
R_{2} & S_{2}
\end{array}\right)=\left(\begin{array}{cc}
1+a_{1} b_{1} \tau & \left(a_{1}+a_{2}\right)+a_{1} b_{1} a_{2} \tau \\
b_{1} & 1+a_{2} b_{1} \tau
\end{array}\right) \\
\left(\begin{array}{ll}
P_{n+1} & Q_{n+1} \\
R_{n+1} & S_{n+1}
\end{array}\right)=\left(\begin{array}{cc}
P_{n}+b_{n} \tau Q_{n} & a_{n+1} P_{n}+\left(1+a_{n+1} b_{n} \tau\right) Q_{n} \\
R_{n}+b_{n} S_{n} & a_{n+1} \tau R_{n}+\left(1+a_{n+1} b_{n} \tau\right) S_{n}
\end{array}\right)
\end{gathered}
$$




$$
\left(\begin{array}{cc}
P_{n+1} & Q_{n+1} \\
R_{n+1} & S_{n+1}
\end{array}\right)=\left(\begin{array}{cc}
P_{n}+b_{n} \tau Q_{n} & a_{n+1} P_{n+1}+Q_{n} \\
R_{n}+b_{n} S_{n} & a_{n+1} \tau R_{n+1}+S_{n}
\end{array}\right) .
$$

The relations (4) and (5) follow from the equality

$$
\left(\begin{array}{cc}
P_{n+1} & \lambda Q_{n+1} \\
\lambda R_{n+1} & S_{n+1}
\end{array}\right)=\left(\begin{array}{cc}
P_{n} & \lambda Q_{n} \\
\lambda R_{n} & S_{n}
\end{array}\right) B^{b_{n}} A^{a_{n+1}} .
$$

In the following sections, we also use the following two relations:

$$
\begin{aligned}
& P_{3}=1+\left(a_{1} b_{1}+a_{1} b_{2}+a_{2} b_{2}\right) \tau+a_{1} b_{1} a_{2} b_{2} \tau^{2} . \\
& Q_{3}= a_{1}+a_{2}+a_{3} \\
&+\left(a_{1} b_{1} a_{2}+a_{1} b_{1} a_{3}+a_{1} b_{2} a_{3}+a_{2} b_{2} a_{3}\right) \tau+a_{1} b_{1} a_{2} b_{2} a_{3} \tau^{2}
\end{aligned}
$$

Using the previous relations we obtain

Proposition 1 The sequences $\left(Q_{n}\right)_{n \geq 1}$ and $\left(Q_{n}\right)_{n \geq 1}$ of polynomials in $\tau$ verify the following identities:

$$
\begin{gathered}
a_{n} Q_{n+1}-\left[\left(a_{n}+a_{n+1}\right)+a_{n} b_{n} a_{n+1} \tau\right] Q_{n}+a_{n+1} Q_{n-1}=0 \\
b_{n} P_{n+1}-\left[\left(b_{n}+b_{n+1}\right)+b_{n} a_{n} b_{n+1} \tau\right] P_{n}+b_{n+1} P_{n-1}=0 .
\end{gathered}
$$

Proof. From (5) we have

$$
P_{n+1}=\frac{1}{a_{n+1}}\left[Q_{n+1}-Q_{n}\right] \text { and } P_{n}=\frac{1}{a_{n}}\left[Q_{n}-Q_{n-1}\right] .
$$

These identities and the equation $P_{n+1}=P_{n}+b_{n} \tau Q_{n}$ give the equation (8). The equation (9) can be similarly obtained .

Let us suppose that $\tau=\frac{p}{q}=\lambda^{2}$ where $p$ and $q$ are non zero integers and $\operatorname{gcd}(p, q)=1$.

If $p=1$ the group $G_{\lambda}$ is not free because in this case $Q_{2}(1,-2 q, 1)(\tau)=0$ (see [1]).

In the following we consider that $p>1$. Then $\mathcal{P}_{\frac{p}{q}}(w) \neq 0$, and $\mathcal{S}_{\frac{p}{q}}(w) \neq 0$. Indeed, if $\mathcal{P}_{\frac{p}{q}}(w) \mathcal{S}_{\frac{p}{q}}(w)=0$ then using the fact that $\operatorname{det}\left(\mathcal{M}_{\tau}(w)\right)=1$ we deduce $-\frac{p}{q} \mathcal{Q}_{\frac{p}{q}}(w) \mathcal{R}_{\frac{p}{q}}(w)=1$ which is in contradiction with the fact that $\operatorname{gcd}(p, q)=1$.

This remark allows us to define a new sequence $\left(x_{n}\right)_{n \geq 1}$ by $x_{n}=\frac{Q_{n}}{P_{n}}$. This sequence satisfies the following relation:

$$
x_{n+1}=a_{n+1}+\frac{1}{b_{n} \tau+\frac{1}{x_{n}}}
$$

Thus we obtain

$$
x_{n+1}=a_{n+1}+\frac{1}{b_{n} \tau+\frac{1}{a_{n}+\frac{1}{b_{n-1} \tau+\frac{1}{a_{n-1}+\cdots}}}} .
$$

These relations are similar with formulas for continued fractions. The properties of these sequences will be used in the next sections of our paper.

Let us also consider the sequence $\left(y_{n}\right)_{n \geq 1}$ defined by: 


$$
y_{n}=y_{n}\left(a_{1}, b_{1}, \cdots, a_{n}, b_{n}\right)=\frac{1}{b_{n} \tau+\frac{1}{x_{n}}}=x_{n+1}-a_{n+1} .
$$

We remark that $x_{n+1}\left(a_{1}, b_{1}, \cdots, a_{n}, b_{n}, a_{n+1}\right)=0$ if and only if

$$
x_{n}\left(a_{1}, b_{1}, \cdots, a_{n}\right)=y_{1}\left(-a_{n+1},-b_{n}\right) .
$$

The following lemma is the key element of Section 5 .

Lemma 1 Let $a_{1}, b_{1}, \cdots, a_{n}, b_{n}, a_{n+1}$ be $2 n+1$ non zero integers and suppose that $\tau>1$. If

$x_{n+1}\left(a_{1}, b_{1}, \cdots, a_{n}, b_{n}, a_{n+1}\right)=0$ then $\left|x_{n}\left(a_{1}, b_{1}, \cdots, a_{n}\right)\right| \leq \frac{1}{\tau-1}$.

Proof. If $x_{n+1}\left(a_{1}, b_{1}, \cdots, a_{n}, b_{n}, a_{n+1}\right)=0$ we have

$$
\left|x_{n}\left(a_{1}, b_{1}, \cdots, a_{n}\right)\right|=\left|y_{1}\left(-a_{n+1},-b_{n}\right)\right|=\frac{1}{\left|b_{n} \tau+\frac{1}{a_{n+1}}\right|} \leq \frac{1}{\left|b_{n}\right| \tau-\frac{1}{\left|a_{n+1}\right|}} \leq \frac{1}{\tau-1} .
$$

Let $\tau=\lambda^{2}$ such that $G_{\lambda}$ is not free. We define the following numerical function:

$\kappa(\tau)=\min \left\{n \in \mathbb{N}^{*}\left|\exists w \in(\mathbb{Z} \backslash\{0\})^{*},\right| w \mid=2 n-1\right.$ and $\left.\mathcal{Q}_{\tau}(w)=0\right\}$. The number $\kappa(\tau)$ will be called the calibre of the group $G_{\lambda}$.

Hence $\kappa(\tau)=2$ if and only if there are non zero integers $a_{1}, b_{1}, a_{2}$ such that $A^{a_{1}} B^{b_{1}} A^{a_{2}}=\left(\begin{array}{ll}* & 0 \\ * & *\end{array}\right)$. Also we have $\kappa(\tau) \leq 3$ if and only if there are non zero integers $a_{1}, b_{1}, a_{2}, b_{2}, a_{3}$ such that $A^{a_{1}} B^{b_{1}} A^{a_{2}} B^{b_{2}} A^{a_{3}}=\left(\begin{array}{ll}* & 0 \\ * & *\end{array}\right)$.

\section{The Diophantine Equation (B1)}

In the next three sections, we consider the following problem $\mathfrak{P}(n)$, where $n \in \mathbb{N}, n \geq 2$ :

- Instance: Two non zero integers $p, q$ with $\operatorname{gcd}(p, q)=1$.

- Question: Is there a word of length $2 n-1$ of non zero integers $w=a_{1} b_{1} \cdots a_{n}$ such that

$$
M_{\tau}(w)=\left(\begin{array}{ll}
* & 0 \\
* & *
\end{array}\right) \text {, where } \tau=\frac{p}{q} ?
$$

So we check solutions in non zero integers $a_{1}, b_{1}, \cdots, a_{n}$ for the diophantine equation

$$
Q_{n}\left(a_{1}, b_{1}, \cdots, a_{n}\right)(\tau)=0 .
$$

The set of $\tau=\lambda^{2}$ for which the Möbius group $G_{\lambda}$ is not free coincides with the set of $\tau$ for which there exists $n \geq 2$ such that the Equation (11) admits solutions.

In this section, we consider the case $n=2$ and in the next section the case $n=3$. The relation $Q_{2}\left(a_{1}, b_{1}, a_{2}\right)=0$ is equivalent to the Equation ( $\left.B^{\prime} 1\right)$ and the relation $Q_{3}\left(a_{1}, b_{1}, a_{2}\right)=0$ is equivalent to the equation ( $\left.B^{\prime} 2\right)$. If $p$ and $q$ are perfect squares we obtain the equations (B1) and (B2).

We will prove that the problem $\mathfrak{P}(2)$ is decidable. The decidability of the problem $\mathfrak{P}(2)$ has already been established by A.F. Beardon (Theorem 2, [2]) for the case when $p$ and $q$ are perfect squares. Our algorithm is simpler and allows us to give an arithmetical criteria for integers $p$ and $q$ for which the problem $\mathfrak{P}(2)$ has solutions (see Theorem 1 below).

First, we prove a result concerning the equation (B'1).

Proposition 2 Let $p$ and $q$ be two integers with $\operatorname{gcd}(p, q)=1$ and $\alpha \in \mathbb{Z}$. Denote

$$
L(p, q, \alpha)=\left\{\left(a_{1}, b_{1}, a_{2}\right) \in\left(\mathbb{Z}^{*}\right)^{3} \mid q\left(a_{1}+a_{2}\right)+p a_{1} b_{1} a_{2}=\alpha\right\} .
$$

Then: 
1. If $\left(a_{1}, b_{1}, a_{2}\right) \in L(p, q, \alpha)$ and $i \in\{1,2\}$ we have

$$
\begin{gathered}
\left|b_{1}\right| \leq \frac{1}{|p|}(|\alpha|+2|q|) \\
\left|a_{i}\right| \leq \frac{1}{|p|}\left(q^{2}+|q|+|p \alpha|\right) .
\end{gathered}
$$

2. The set $L(p, q, \alpha)$ is finite.

Proof.

1) Let $\left(a_{1}, b_{1}, a_{2}\right) \in L(p, q, \alpha)$ and for $i \in\{1,2\}$ put $d_{i}=q+p a_{i} b_{1}$. Then $d_{1} d_{2}=q^{2}+p b_{1} \alpha$, $d_{i} \equiv q(\bmod p)$ and $d_{i} \neq q$.

As $b_{1}=\frac{1}{p}\left[\frac{\alpha}{a_{1} a_{2}}-q\left(\frac{1}{a_{1}}+\frac{1}{a_{2}}\right)\right]$ we deduce $\left|b_{1}\right| \leq \frac{1}{|p|}(|\alpha|+2|q|)$. Because $\left|d_{i}\right| \leq q^{2}+\left|p b_{1} \alpha\right|$ and $a_{i}=\frac{d_{i}-q}{p b_{1}}$ we have

$$
\left|a_{i}\right| \leq \frac{\left|d_{i}\right|+|q|}{\left|p b_{1}\right|} \leq \frac{1}{|p|}\left(q^{2}+|q|+|p \alpha|\right) .
$$

2) results from (1).

Using the previous proposition we can obtain the decidability of the problem $\mathfrak{P}(2)$.

Theorem 1 Let $p$ and $q$ be two integers with $\operatorname{gcd}(p, q)=1$. The following sentences are equivalent:

1. The equation $q\left(a_{1}+a_{2}\right)+p a_{1} b_{1} a_{2}=0$ has solutions in non zero integers.

2. There exists a divisor $d$ of $q^{2}, d \neq q$ such that $d \equiv q(\bmod p)$.

3. $\tau=\frac{p}{q} \in\left\{\frac{1}{m}+\frac{1}{n} \mid(m, n) \in\left(\mathbb{Z}^{*}\right)^{2}, m+n \neq 0\right\}$.

Proof. The equivalence between (1) and (2) results from the Proposition 2. It is enough to consider $\alpha=0$ in that proposition. The equivalence between (1) and (3) is obvious.

Remark 1 Let $D(n)$ be the set of all divisors of the integer $n$. If $d$ is like in (2) of the previous Theorem 1 then a solution $\left(a_{1}, b_{1}, a_{2}\right)$ to the equation ( $\left.B^{\prime} 1\right)$ can be obtained by taking

- $b_{1} \in D\left(\frac{d_{1}-q}{p}\right) \cap D\left(\frac{d_{2}-q}{p}\right)$ and

- $a_{i}=\frac{d_{i}-q}{p b_{1}}$ for $i \in\{1,2\}$

where $d_{1}=d$ and $d_{2}=\frac{q^{2}}{d}$. Moreover any solution $\left(a_{1}, b_{1}, a_{2}\right)$ of the equation $\left(B^{\prime} 1\right)$ can be obtained by this method. We can write $\tau$ as in (3) of the Theorem 1

$$
\tau=\frac{p}{q}=\frac{1}{\frac{q-d_{1}}{p}}+\frac{1}{\frac{q-d_{2}}{p}} .
$$

The results of A.F. Beardon ([2], theorem 2) concerning the problem $\mathfrak{P}(2)$ for the case when $p$ and $q$ are perfect squares (or equivalently when $\lambda \in \mathbb{Q}$ ) result immediately from the next corollary.

Corollary 1 Let $p$ and $q$ be two non zero integers with $\operatorname{gcd}(p, q)=1$ and $\tau=\frac{p^{2}}{q^{2}}=\lambda^{2}$. The group

$G_{\lambda}$ is not free with the calibre $k(\tau)=2$ if and only if there exists a divisor $d$ of $q^{4}, d \neq q^{2}$ such that $d \equiv q^{2}\left(\bmod p^{2}\right)$.

From the previous theorem it also follows: 
1) The equation ( $\left.B^{\prime} 1\right)$ has no solution if $\tau=\frac{p}{q}>2$.

2) $k\left(\frac{p}{q}\right)=2$ in the following cases: a) $p=1$; b) $p=2$; c) $p \geq 3$ and $q=k p \pm 1$ with $k \in \mathbb{N}^{*}$.

Below we present another form of the Theorem 1 in which we use the decomposition of $q$ as a product of prime numbers.

Theorem 2 Let $p$ and $q$ be two integers with $p>2$ and $\operatorname{gcd}(p, q)=1$. Let us suppose that the decomposition of $q$ as a product of powers of distinct prime numbers $\pi_{1}, \pi_{2}, \cdots, \pi_{m}$ is $q=\pi_{1}^{\alpha_{1}} \pi_{2}^{\alpha_{2}} \cdots \pi_{m}^{\alpha_{m}}$.

Then $k\left(\frac{p}{q}\right)=2$ if and only if there exist:

- two disjoint subsets $I$ and $J$ of $\{1,2, \cdots, m\}$ with $I \cup J \neq \varnothing$.

- a set of integers $\left(\delta_{l}\right)_{l \in I \cup J}$ with $1 \leq \delta_{l} \leq \alpha_{l}$ for every $l \in I \cup J$.

- $\varepsilon \in\{1,-1\}$.

such that $\prod_{i \in I} \pi_{i}^{\delta_{i}} \equiv \varepsilon \prod_{j \in J} \pi_{j}^{\delta_{j}}(\bmod p)$.

Proof. Let $d=\varepsilon \pi_{1}^{\beta_{1}} \pi_{2}^{\beta_{2}} \cdots \pi_{m}^{\beta_{m}}$ be a divisor of $q^{2}, d \neq \pm q$. We can drop the case $d=-q$ because $p>2$. Hence $\left(\beta_{1}, \beta_{2}, \cdots, \beta_{m}\right) \neq\left(\alpha_{1}, \alpha_{2}, \cdots, \alpha_{m}\right)$ and $0 \leq \beta_{k} \leq 2 \alpha_{k} \quad$ for every $k \in\{1,2, \cdots, m\}$. We put $I=\left\{i \in\{1,2, \cdots, m\} \mid \beta_{i}<\alpha_{i}\right\}$ and $J=\left\{j \in\{1,2, \cdots, m\} \mid \beta_{j}>\alpha_{j}\right\}$. Then $I \cap J=\varnothing$ and $I \cup J \neq \varnothing$. Let:

$$
\delta_{\ell}=\left\{\begin{array}{ll}
\alpha_{\ell}-\beta_{\ell} & \text { if } \ell \in I \\
\beta_{\ell}-\alpha_{\ell} & \text { if } \ell \in J
\end{array} .\right.
$$

We have $1 \leq \delta_{\ell} \leq \alpha_{\ell}$ for every $\ell \in I \cup J$. The condition $d \equiv q(\bmod p)$ is equivalent to

$$
\prod_{i \in I} \pi_{i}^{\delta_{i}} \equiv \varepsilon \prod_{j \in J} \pi_{j}^{\delta_{j}}(\bmod p) .
$$

Corollary 2 Let $p$ and $\alpha$ be two non zero integers and $\pi$ be a prime number. Suppose that $\operatorname{gcd}(p, \pi)=1$. Then $k\left(\frac{p}{\pi^{\alpha}}\right)=2$ if and only if there exists an integer $\delta$ with $1 \leq \delta \leq \alpha$ such that $\pi^{\delta} \equiv \varepsilon(\bmod p)$ where $\varepsilon \in\{1,-1\}$.

Proof. We take $m=1$ in the previous theorem.

Example: Using the previous results and an example from ([7]) we have $k\left(\frac{12}{127}\right)=3$ and $k\left(\frac{12}{127^{2}}\right)=2$.

\section{The Beardon Diophantine Equation (B2)}

Now we consider the problem $\mathfrak{P}(3)$. We mention that the equation $Q_{3}\left(a_{1}, b_{1}, a_{2}, b_{2}, a_{3}\right)(\tau)=0$ has been considered in several papers (see [2] [8] [10]) for the case when $p$ and $q$ are perfect squares.

From now on, we suppose that $Q_{2}\left(a_{1}, b_{1}, a_{2}\right)(\tau) \neq 0$ for every $\left(a_{1}, b_{1}, a_{2}\right) \in\left(\mathbb{Z}^{*}\right)^{3}$ i.e. following Theorem 1 , $\tau$ does not belong to $A=\left\{\frac{1}{m}+\frac{1}{n} \mid(m, n) \in\left(\mathbb{Z}^{*}\right)^{2}, m+n \neq 0\right\}$. Hence we can define a function $\varphi:] 0,4\left[\cap(\mathbb{Q} \backslash A) \rightarrow \mathbb{Q}_{+}^{*}\right.$ by $\varphi(\tau)=\inf \{\mid \tau-\alpha \| \alpha \in A\}$. We remark that $\varphi(\tau)>0$ and

a) $\varphi(\tau)=\tau-2$ if $\tau \in] 2,4[$.

b) $\varphi(\tau)=\min \left\{\tau-1-\frac{1}{k+1}, 1+\frac{1}{k}-\tau\right\}$ if $\left.\tau \in\right] 1,2\left[\right.$, where $k=\left[\frac{1}{\tau-1}\right]$.

Using the relations (8) for the sequence of polynomials $\left(Q_{n}\right)_{n \geq 1}$ we prove that the problem $\mathfrak{P}(3)$ is decidable.

Theorem 3 Let $\tau \in Q \cap] 0,4\left[\right.$ such that $\tau$ does not belong to the set $\left\{\frac{1}{m}+\frac{1}{n} \mid(m, n) \in\left(\mathbb{Z}^{*}\right)^{2}\right\}$. Then the 
equation

$$
Q_{3}\left(a_{1}, b_{1}, a_{2}, b_{2}, a_{3}\right)(\tau)=0
$$

has a finite number of solutions $\left(a_{1}, b_{1}, a_{2}, b_{2}, a_{3}\right) \in\left(\mathbb{Z}^{*}\right)^{5}$.

Proof. Using the relations (8) we deduce that

$$
Q_{2}\left(a_{1}, b_{1}, a_{2}\right) Q_{2}\left(a_{2}, b_{2}, a_{3}\right)=a_{1} a_{3} .
$$

Hence $\left(\tau+\frac{1}{b_{1}}\left(\frac{1}{a_{1}}+\frac{1}{a_{2}}\right)\right)\left(\tau+\frac{1}{b_{2}}\left(\frac{1}{a_{2}}+\frac{1}{a_{3}}\right)\right)=\frac{1}{b_{1} b_{2} a_{2}^{2}}$. Using the function $\varphi$ we have:

$$
\left|b_{1} b_{2}\right| a_{2}^{2} \leq \frac{1}{[\varphi(\tau)]^{2}} .
$$

We obtain a finite number of possibilities for $b_{1}, b_{2}$ and $a_{2}$. So $a_{1}$ and $a_{3}$ remain to be studied. From the equation

$$
Q_{3}\left(a_{1}, b_{1}, a_{2}, b_{2}, a_{3}\right)(\tau)=0
$$

it follows that

$$
P_{3}\left(a_{1}, b_{1}, a_{2}, b_{2}, a_{3}\right) S_{3}\left(a_{1}, b_{1}, a_{2}, b_{2}, a_{3}\right)=1 .
$$

Hence there exists $\left(d_{1}, d_{2}\right) \in \mathbb{Z}^{2}$ such that

- $d_{1} d_{2}=q^{4}$.

- $q^{2}+\left(a_{1} b_{1}+a_{1} b_{2}+a_{2} b_{2}\right) q p+a_{1} b_{1} a_{2} b_{2} p^{2}=d_{1}$.

- $q^{2}+\left(a_{2} b_{2}+a_{3} b_{1}+a_{2} b_{1}\right) q p+a_{3} b_{2} a_{2} b_{1} p^{2}=d_{2}$.

Thus there exists a finite number of possibilities for $a_{1}$ and $a_{3}$.

If $Q_{3}\left(a_{1}, b_{1}, a_{2}, b_{2}, a_{3}\right)(\tau)=0$ from the inequality $\left|b_{1} b_{2}\right| a_{2}^{2} \leq \frac{1}{[\varphi(\tau)]^{2}}$ we obtain

a) If $\tau \in] 3,4[$ then $\mathfrak{P}(3)$ has no solution.

b) If $\tau \in] 2+\frac{1}{\sqrt{2}}, 3\left[\right.$ then $b_{1}, b_{2}, a_{2} \in\{-1,1\}$.

We also remark that the equation $\left(B^{\prime} 2\right)$ is equivalent to the following equation

$$
\frac{a_{1} q}{q+a_{1} b_{1} p}+\frac{a_{3} q}{q+a_{3} b_{2} p}=-a_{2} .
$$

This enables us to obtain some explicit expressions for the rationals $\tau$ such that equation (B'2) has solutions in $\mathbf{Z}^{*}$.

Proposition 3 Let $k, \ell$ be two non zero integers and $k_{1}, k_{2}$ be two divisors of $k$. If $\tau=\frac{1}{k}+\frac{1}{\ell}\left(\frac{1}{k_{1}}+\frac{1}{k_{2}}\right)$ then the equation (B'2) has solutions in $\mathbf{Z}^{*}$.

Proof. Let $b_{1}=k_{1}, b_{2}=k_{2}, a_{2}=-\ell$ and $b_{1} a_{1}=b_{2} a_{3}=-k$. Then (10) is equivalent to $\tau=\frac{1}{k}+\frac{1}{\ell}\left(\frac{1}{k_{1}}+\frac{1}{k_{2}}\right)$.

Note that if in equation ( $\left.B^{\prime} 2\right)$ we have $b_{1} a_{1}=b_{2} a_{3}$ then $\tau$ is exactly given by the above expression.

Using once again (10) we obtain

Proposition 4 Let $\alpha$ and $\alpha^{\prime}$ be in $\mathbf{Z}^{*}$ with $|\alpha| \neq\left|\alpha^{\prime}\right|$. If

$$
\tau=\frac{\alpha^{2}+\alpha^{\prime 2}}{\alpha \alpha^{\prime}\left(\alpha-\alpha^{\prime}\right)}
$$

then the equation $\left(B^{\prime} 2\right)$ has solutions in $\mathbf{Z}^{*}$. 
Proof. Consider (10) for $b_{1}=b_{2}=1, a_{1}=\alpha$ and $a_{3}=-\alpha^{\prime}$. Then $\frac{1}{b_{1} \tau+\frac{1}{a_{1}}}=\alpha-\rho$ and $\frac{1}{b_{2} \tau+\frac{1}{a_{3}}}=-\alpha^{\prime}+\rho$, where $\rho=\frac{\alpha^{2}+\alpha^{\prime 2}}{\alpha+\alpha^{\prime}}$. It follows that if we take $-a_{2}=\alpha-\alpha^{\prime}$ then (10) is verified.

In the next proposition we give another method to obtain solutions of Equation ( $\left.B^{\prime} 2\right)$. It is similar to those presented in [8] and [10].

Proposition 5 Let $p$ and $q$ be two integers with $\operatorname{gcd}(p, q)=1$. Suppose that there exist $a_{1}, b_{2}$ and $a_{2}$ in $\mathbf{Z}^{*}$ such that $\left(a_{1}+a_{2}\right) q+a_{1} b_{1} a_{2} p=1$. If $\tau=\frac{p}{q}$ then the equation (B'2) has solutions in $\mathbf{Z}^{*}$.

Proof. Let $A_{1}=a_{1} q, B_{1}=b_{1} a_{2}, A_{2}=-1, B_{2}=-b_{2} a_{1}$ and $A_{3}=a_{2} q$. Then $\frac{1}{B_{1} \tau+\frac{1}{A_{1}}}+\frac{1}{B_{2} \tau+\frac{1}{A_{2}}}=-A_{2}$. Hence the equation (B'2) has solutions.

We end this section with the following open questions:

Questions:

1) Find all the solutions of (B2).

2) Find arithmetical characterizations (similar to those given in Theorem 1 for the positive integers $p$ and $q$ for which the problem $\mathfrak{P}(3)$ has solutions.

\section{Increasing Unbounded Lower Bound Function for $\mathrm{K}$}

In this section, we prove that in order to show that the group $G_{\lambda}$ is not free for a rational $\tau$ with $\tau=\lambda^{2}<4$ and $\tau$ close to 4 , we have to consider longer and longer words in $A$ and $B$. Similar remarks (without any proof) have been made by A.F. Beardon in [2] and S.P. Farbman in [7].

Everywhere in this section, we consider that $\tau$ is a rational number in the open interval ]2,4[ .

From the Lemma 1, Section 2, if $x_{n+1}\left(a_{1}, \cdots, a_{n}, b_{n}, a_{n+1}\right)(\tau)=0$ then $\left|x_{n}\left(a_{1}, \cdots, a_{n}\right)(\tau)\right| \leq \frac{1}{\tau-1}$. For this reason we consider the sequence $\left(\alpha_{n}\right)_{n \geq 1}$ of rational functions in the variable $\tau, \alpha_{n}=\alpha_{n}(\tau)$, defined by:

$$
\left\{\begin{array}{l}
\alpha_{1}=1 \\
\alpha_{n+1}=1-\frac{1}{\tau-\frac{1}{\alpha_{n}}}
\end{array}\right.
$$

For example

$$
\alpha_{2}(\tau)=\frac{\tau-2}{\tau-1}, \alpha_{3}(\tau)=\frac{\tau^{2}-4 \tau+3}{\tau^{2}-3 \tau+1} \text { and } \alpha_{4}(\tau)=\frac{\tau^{3}-6 \tau^{2}+10 \tau-4}{\tau^{3}-5 \tau^{2}+6 \tau-1} .
$$

We also define the function $l:] 2,4[\rightarrow \mathbf{N} \backslash\{0,1\}$ by the formula:

$$
l(\tau)=\inf \left\{k \in \mathbf{N}^{*} \mid \alpha_{k}(\tau) \leq \frac{1}{\tau-1}\right\} .
$$

Thus one has $l(\tau)=2$ if and only if $\tau \in] 2,3], l(\tau)=3$ if and only if $\tau \in] 3,2+\sqrt{2}]$ and $l(\tau)=4$ if and only if $\left.\tau \in] 2+\sqrt{2}, \frac{5+\sqrt{5}}{2}\right]$.

Now we will calculate $\alpha_{n}(\tau)$.

Note that $\alpha_{n}(\tau)=x_{n}(1,-1,1,-1, \cdots,-1,1)(\tau)$. For this reason we find the matrix 
$X_{n+1}=A B^{-1} A B^{-1} \cdots A B^{-1} A=\left(A B^{-1}\right)^{n} A=C^{n} A$, where $C=A B^{-1}$. We suppose now that $\lambda=2 \sin \left(\frac{\theta}{2}\right)$ with $\theta \in] \frac{\pi}{2}, \pi\left[\right.$, so $\tau=\lambda^{2}=2(1-\cos \theta)$. As $\operatorname{trace}(C)=2-\tau=2 \cos \theta$ the matrix $C$ verifies the equation:

$$
C^{2}-2 \cos \theta C+I_{2}=O_{2} \text {. }
$$

Using this relation we find that

$$
C^{n}=\frac{1}{\sin \theta}\left(\begin{array}{cc}
\sin ((n+1) \theta)-\sin \theta & 2 \sin \left(\frac{\theta}{2}\right) \sin (n \theta) \\
-2 \sin \left(\frac{\theta}{2}\right) \sin (n \theta) & \sin (n \theta)-\sin \left((n-1) \frac{\theta}{2}\right)
\end{array}\right) .
$$

Hence $\alpha_{n+1}(\tau)=\frac{\sin ((n+1) \theta)}{\sin ((n+1) \theta)-\sin (n \theta)}=\frac{1}{1-\frac{\sin (n \theta)}{\sin ((n+1) \theta)}}$.

Lemma 2 Let $(\alpha, \tau, x) \in \mathbb{R}^{3}, \tau>2$ be such that $|x| \geq \alpha>\frac{1}{\tau-1}$. Then for every $a, b \in \mathbf{Z}^{*}$ we have $\left|a+\frac{1}{b \tau+\frac{1}{x}}\right| \geq 1-\frac{1}{\tau-\frac{1}{\alpha}}>0$.

Proof. Since $\frac{1}{|x|} \leq \tau-1<\tau$ we obtain that $\left|\frac{1}{b \tau+\frac{1}{x}}\right| \leq \frac{1}{\tau-\frac{1}{\alpha}}<1$. Hence $\left|a+\frac{1}{b \tau+\frac{1}{x}}\right| \geq 1-\frac{1}{\tau-\frac{1}{\alpha}}$.

The previous expression for $\alpha_{n}(\tau)$ and Lemma 2 show that $l(\tau)$ is well defined and $l(\tau)<\kappa(\tau)$, for every $\tau$ in the open interval $] 2,4[$. So $l$ is a lower bound numerical function for the function $\kappa$ restricted to $] 2,4[$.

Theorem 4 For any $n \in \mathbb{N}^{*}$ and $\left.\tau \in\right] 2,4[$ one has $l(\tau)=n+1$ if and only if there exists

$\left.\theta \in] \frac{n}{n+1} \pi, \frac{n+1}{n+2} \pi\right]$ such that $\tau=2-2 \cos \theta$.

Proof. Let $l(\tau)=n+1$, where $n \in \mathbb{N}^{*}$. From the definition of the function $l$ this previous equality holds if and only if $\alpha_{n+1}(\tau) \leq \frac{1}{\tau-1}$ and $\alpha_{k}(\tau)>\frac{1}{\tau-1}$, for all $k \in\{1, \cdots, n\}$. But $\alpha_{k}(\tau)>\frac{1}{\tau-1}$ if and only if $\frac{1}{1-\frac{\sin ((k-1) \theta)}{\sin (k \theta)}}>\frac{1}{1-2 \cos \theta}$.

Thus we obtain the system of two inequalities $\frac{\sin ((k-1) \theta)}{\sin (k \theta)}<1$ and $\frac{\sin ((k+1) \theta)}{\sin (k \theta)}<0$.

Finally, $l(\tau)=n+1$ if and only if we have $\frac{\sin ((n+1) \theta)}{\sin ((n+2) \theta)} \geq 0$ and $\frac{\sin (k \theta)}{\sin ((k+1) \theta)}<0$ for all $k \in\{1, \cdots, n\}$.

These inequalities give $\left.\theta \in] \frac{n}{n+1} \pi, \frac{n+1}{n+2} \pi\right]$.

Corollary 3 The function $l$ is increasing and unbounded. 
Therefore

$$
\lim _{\tau \rightarrow 4, \tau<4} l(\tau)=\lim _{\tau \rightarrow 4, \tau<4} \kappa(\tau)=\infty
$$

Example: We consider the sequence $\tau_{n}=4-\frac{1}{2^{n}}$, for $n \in \mathbf{N}$.

- For $n=0$ we have $\tau_{0}=3$. So $\alpha_{2}\left(\tau_{0}\right)=\frac{1}{2} \leq \frac{1}{\tau_{0}-1}$, hence $l\left(\tau_{0}\right)=2$. As $x_{3}(-1,1,-1,1,-1)\left(\tau_{0}\right)=0$, it follows that $\kappa\left(\tau_{0}\right)=3$.

- For $n=1$ we have $\tau_{1}=\frac{7}{2}$ and $\alpha_{2}\left(\tau_{1}\right)=\frac{3}{5}, \alpha_{3}\left(\tau_{1}\right)=\frac{5}{11}, \alpha_{4}\left(\tau_{1}\right)=\frac{3}{13}<\frac{1}{\tau_{1}-1}=\frac{2}{5}$. Hence $l\left(\tau_{1}\right)=4$ and since

$$
x_{5}(2,-1,1,-1,1,-1,1,-1,2)\left(\tau_{1}\right)=0
$$

we have $\kappa\left(\tau_{1}\right)=5$

- For $n=2$ we have $\tau_{2}=\frac{15}{4}$ and $\alpha_{2}\left(\tau_{2}\right)=\frac{7}{11}, \quad \alpha_{3}\left(\tau_{2}\right)=\frac{33}{61}, \quad \alpha_{4}\left(\tau_{2}\right)=\frac{119}{251}, \quad \alpha_{5}\left(\tau_{2}\right)=\frac{305}{781}$, $\alpha_{6}\left(\tau_{2}\right)=\frac{231}{451}<\frac{1}{\tau_{2}-1}=\frac{4}{11}$. Hence $l\left(\tau_{2}\right)=6$ and $\kappa\left(\tau_{2}\right) \geq 7$. From [7] we have $\kappa\left(\tau_{2}\right) \leq 18$.

\section{Questions:}

1) Is it true that for every $\tau \in \mathbb{Q} \cap] 0,4[$ and $n \in \mathbb{N}, n \geq 2$, the problem $\mathfrak{P}(n)$ is decidable?

2) Is it true that for every $\tau \in \mathbb{Q} \cap] 0,4[$ there exists $n \in \mathbb{N}, n \geq 2$ such that the problem $\mathfrak{P}(n)$ is decidable?

3) Is it true that for every $\tau \in \mathbb{Q} \cap] 0,4[$ there exists $n \in \mathbb{N}, n \geq 2$, such that the problem $\mathfrak{P}(n)$ has solutions?

4) Find $\kappa\left(\tau_{n}\right)$, for $n \geq 2$.

\section{Acknowledgements}

I thank Elias Tahhan (University S. Bolivar, Caracas) and Jerzy Tomasik (Universite d'Auvergne, ClermontFerrand) for discussion concerning some logical aspects of my paper.

\section{References}

[1] Lyndon, R.C. and Ullman, J.L. (1969) Groups Generated by Two Linear Parabolic Transformations. Canadian Journal of Mathematics, 21, 1388-1403. http://dx.doi.org/10.4153/CJM-1969-153-1

[2] Beardon, A.F. (1993) Pell's Equation and Two Generator Möbius Groups. Bulletin of the London Mathematical Society, 25, 527-532. http://dx.doi.org/10.1112/blms/25.6.527

[3] Klarner, D., Birget, J.-C. and Satterfield, W. (1991) On the Undecidability of the Freeness of Integer Matrix Semigroups. International Journal of Algebra and Computation, 1, 223-226. http://dx.doi.org/10.1142/S0218196791000146

[4] Cassaigne, J., Harju, T. and Karhumaki, J. (1999) On the Undecidability of the Freeness of Matrix Semigroups. International Journal of Algebra and Computation, 9, 295-305. http://dx.doi.org/10.1142/S0218196799000199

[5] Cassaigne, J. and Nicolas, F. (2012) On the Decidability of Semigroup Freeness. RAIRO_Theoretical Informatics and Applications, 46, 355-399. http://dx.doi.org/10.1051/ita/2012010

[6] Gawrychowski, P., Gutan, M. and Kisielewicz, A. (2010) On the Problem of Freness of Multplicative Matrix Semigroups. Theoretical Computer Science, 411, 1115-1120. http://dx.doi.org/10.1016/j.tcs.2009.12.005

[7] Farbman, S.P. (1995) Non-Free Two-Generator Subgroups of $S L_{2}(Q)$. Publicacions Mathemàtiques, 39, $379-391$. http://dx.doi.org/10.5565/PUBLMAT_39295_13

[8] Tan, E.-C. and Tan, S.-P. (1996) Quadratic Diophantine Equations and Two Generators Möbius Groups. Journal of the Australian Mathematical Society, 61, 360-368. http://dx.doi.org/10.1017/S1446788700000434

[9] de la Harpe, P. (2000) Topics in Geometric Group Theory. Chicago Lectures in Mathematics. University of Chicago Press, Chicago. 
[10] Grytczuk, A. and Wojtowicz, M. (2000) Beardon’s Diophantine Equations and Non-Free Möbius Groups. Bulletin of the London Mathematical Society, 32, 305-310. http://dx.doi.org/10.1017/S1446788700000434

[11] Bamberg, J. (2000) Non-Free Points for Groups Generated by a Pair of $2 \times 2$ Matrices. Journal of the London Mathematical Society, 62, 795-801. http://dx.doi.org/10.1112/S0024610700001630 